\title{
The Paterno-Büchi Reaction as a Demonstration of Chemical Kinetics and Synthetic Photochemistry Using Light Emitting Diode Apparatus \\ DOI:
}

10.1021/acs.jchemed.5b00129

\section{Document Version}

Accepted author manuscript

Link to publication record in Manchester Research Explorer

Citation for published version (APA):

Thompson, M. P., Agger, J., \& Wong, L. S. (2015). The Paterno-Büchi Reaction as a Demonstration of Chemical Kinetics and Synthetic Photochemistry Using Light Emitting Diode Apparatus. Journal of Chemical Education, 92(10), 1716. https://doi.org/10.1021/acs.jchemed.5b00129

Published in:

Journal of Chemical Education

\section{Citing this paper}

Please note that where the full-text provided on Manchester Research Explorer is the Author Accepted Manuscript or Proof version this may differ from the final Published version. If citing, it is advised that you check and use the publisher's definitive version.

\section{General rights}

Copyright and moral rights for the publications made accessible in the Research Explorer are retained by the authors and/or other copyright owners and it is a condition of accessing publications that users recognise and abide by the legal requirements associated with these rights.

\section{Takedown policy}

If you believe that this document breaches copyright please refer to the University of Manchester's Takedown Procedures [http://man.ac.uk/04Y6Bo] or contact uml.scholarlycommunications@manchester.ac.uk providing relevant details, so we can investigate your claim.

\section{OPEN ACCESS}




\section{The Paternò-Büchi Reaction as a Demonstration of Chemical Kinetics and Synthetic Photochemistry Using a Light Emitting Diode Apparatus.}

$\dagger$ Manchester Institute of Biotechnology, University of Manchester, 131 Princess Street, Manchester M1 7DN, United Kingdom.

$\ddagger$ School of Chemistry, University of Manchester, Oxford Road, Manchester M13 9PL, United Kingdom.

\section{ABSTRACT}

The Paternò-Büchi photocycloaddition reaction is used as the basis for physical-organic final-year undergraduate laboratory experiments designed to emphasize the multidisciplinary approach to modern-day chemical practice. These reactions are performed using commercially available LED-based light sources, which offer a convenient and safe tool for teaching photochemistry. Using a series of substituted benzaldehydes and furan, experiments can be conducted to measure reaction rates and to isolate the products for structure determination. The experiments are deliberately broad in scope, covering mechanistic photochemistry, chemical kinetics and photochemical organic synthesis in a holistic manner in order to demonstrate to students how topics usually taught separately are brought together. The reported experimental program also offers actinometry and quantum yield determinations, as well as NMR-based structural analysis, as potential routes for further elaboration of the experimental program. 


\section{ABSTRACT GRAPHIC}

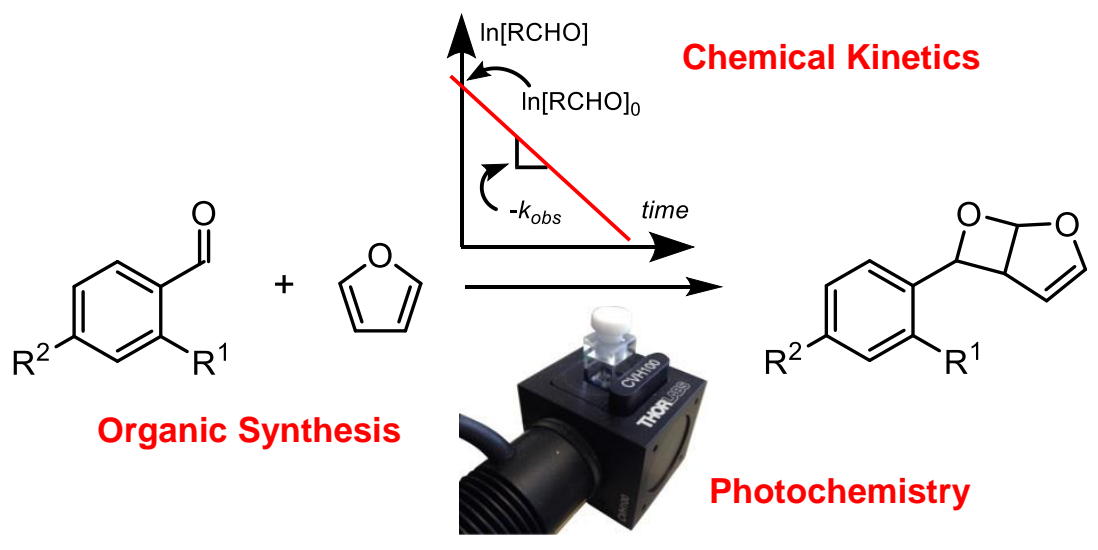

\section{KEYWORDS}

upper-division undergraduate, organic chemistry, physical chemistry, mechanisms of reaction, laboratory instruction, kinetics, rate law, laboratory equipment/apparatus, photochemistry, collaborative/cooperative learning 


\section{INTRODUCTION}

Although a large number of photochemical organic transformations have been reported in the research literature, including as key steps towards the synthesis of natural products, ${ }^{1}$ the true potential of photochemistry has not yet been fully exploited. This aversion to photochemistry appears to be due to a general lack of familiarity and the view that it is experimentally inconvenient or unselective. ${ }^{1 \mathrm{~b}, 2}$ Typically, high-power, omnidirectional Hg lamps (usually with water cooling) are used, which carry considerable practical and safety implications. However, the availability of light-emitting diode (LED) technology now offers a means to address these shortcomings, especially in the context of an undergraduate laboratory where reliability, robustness and safety are key considerations. LEDs are entirely solid-state and therefore lack the fragility of classical glass lamps. Their low heat generation also means that passive cooling is sufficient. They are able to provide variable power, directional, near monochromatic light (typically $7.5 \mathrm{~nm}$ full width half maximum at $365 \mathrm{~nm}$ ) at essentially any desired wavelength, therefore enabling highly selective reactions.

The archetypal example of organic photochemistry for undergraduates is the $E, Z$ isomerisation of olefins. ${ }^{3}$ However, these reactions are synthetically limited in scope and there are relatively few examples of reactions that are relevant for modern synthetic chemistry, ${ }^{2 \mathrm{~b}}$ such as photocycloadditions. The development of a photocycloaddition reaction in a laboratory education setting offers the opportunity for students to contextualise their knowledge of spectroscopy (analytical chemistry), kinetics (physical chemistry) and mechanism (organic chemistry) in a holistic manner that combines subjects normally taught separately.

In reviewing the organic photochemistry literature, the Paternò-Büchi (PB) reaction was identified as a suitable candidate for development into an undergraduate laboratory experiment. This reaction involves the [2+2] cycloaddition of a carbonyl group and an alkene to yield four-membered oxetane rings (Scheme 1). This reaction has not 
previously been reported in chemical education literature despite being one of the most widely studied and applied photochemical reactions. ${ }^{4}$ It was considered to be particularly suitable in this case because:

- it can be highly selective (forms a single product); ${ }^{5}$

- its mechanism has been well studied and can be rationalised from undergraduate-level knowledge of photochemistry, organic reaction mechanisms and kinetics;6

- the wavelength of light required to drive the reaction is $\sim 365 \mathrm{~nm}$, simultaneously avoiding the use of more hazardous short wavelength UV light while being essentially unaffected by standard laboratory lighting; quantified by UV-Vis spectrophotometry.

Scheme 1. General Scheme for the PB Reaction

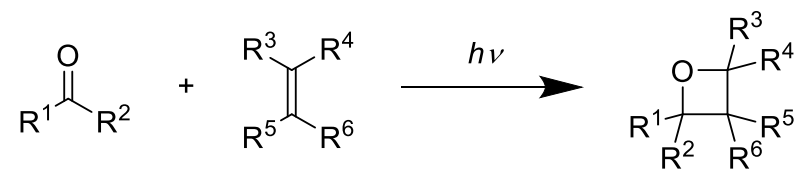

This report details the implementation of the PB reaction in the context of undergraduate laboratory education and the use of LED-based apparatus as a convenient tool for the teaching of photochemistry. Here, the reaction of three aldehydes with furan gives highly reproducible results and are of sufficient structural complexity to demonstrate a range of organic chemistry topics. The pedagogic goals for this experimental programme are to:

- introduce the use of photochemistry in practical organic synthesis;

- introduce the use of LEDs as a convenient tool to perform photochemistry; 
- demonstrate how organic, physical and analytical topics are applied in the context of an experimental programme;

- provide practice in the collection and interpretation of analytical data. As an upper-level undergraduate experiment, the emphasis is on instilling analytical rigor and critical assessment of the results (i.e., how to turn data into insight).

Thus far, this experiment has been completed by 28 students in a synthetic chemistry laboratory course on three occasions.

\section{EXPERIMENTAL}

The photolysis apparatus is assembled using mounted high-power LEDs emitting at $365 \mathrm{~nm}$ connected to a cuvette holder (Thorlabs, NJ). The LED is powered by a driver providing a constant current of $700 \mathrm{~mA}$, corresponding to a nominal LED power output of $190 \mathrm{~mW}$. UV-Vis spectra are recorded using a UV-Vis-NIR spectrophotometer with quartz cuvettes. Detailed instructions for the assembly of the photolysis apparatus, preparation of stock solutions and experimental procedures are provided in the Supporting Information.

The students conduct the practical experimentation individually, but at the start of the program each is assigned a specified amount of furan that they should use for the PB reactions. Each student calculates the observed rate constant for their assigned amount of furan, but then combine their data to calculate the overall rate constant. This experimental program is divided into five, two-day sessions and spread over a five week period (i.e., two consecutive days per week). The entire program can be run over ten consecutive days, or any combination of two-day sessions, but individual sessions should not be split because some of the reactions require overnight irradiation followed by workup the following day. All of the PB reactions used in this experimental program involve the reaction of one of the benzaldehydes 2-4 with furan (1) to give the 
corresponding oxetanes 5-7 as single products (Scheme 2). In each laboratory session, all students use the same aldehyde. The experiments pertaining to the PB reactions, therefore, only occupy three sessions (i.e., one for each aldehyde). The remaining two sessions are devoted to chemical actinometry, to determine the photon flux delivered by the apparatus (required for quantum yield calculations), and data analysis involving the plotting of the relevant graphs and extraction of the rate constants.

\section{Scheme 2. PB Reaction between Furan (1) and Aromatic Aldehydes (2-4) used in this Laboratory Experiment}<smiles>[R]c1ccc(C=O)c([R])c1</smiles>

toluene<smiles>[R]c1ccc(C2OC3OC=CC32)c([R])c1</smiles>

oxetane

5

7

In a typical laboratory session, the first day involves time-dependent measurements of a PB reaction. Each student is assigned a different, but known, amount of $\mathbf{1}$ to react with the chosen aldehyde for that session. The consumption of the aldehyde is measured by UV-Vis spectrophotometry $(350 \mathrm{~nm})$ of the reaction mixture, and students use these data to plot a time course for the experiment, calculate the observed rate constant, $k_{o b s}$, and differential quantum yield, $\Phi$.

In order to achieve reasonable linear correlations and convenient experimental execution, it is recommended that an aldehyde starting concentration of $0.004 \mathrm{M}$ and between $0.04-0.29 \mathrm{M}$ of furan is used. This mixture is placed in a quartz cuvette and irradiated in the photolysis apparatus. At specified time intervals, $t$ (between $5-120$ min), the UV-Vis absorbance is recorded. As each student is using a different quantity 
of furan, all students pool their data to calculate the actual rate constant $(k)$ from the $k_{\text {obs }}$ they had previously individually determined.

At the end of the first day, students perform a larger-scale PB experiment that is allowed to progress overnight. On the second day, students isolate and fully characterise the oxetane product using MS, NMR and IR spectroscopy, thereby providing training in (semi)preparative-scale organic photochemistry.

\section{HAZARDS}

As with any chemistry experiment, students must apply Good Laboratory Practice and wear appropriate protective equipment. The PB reaction employs UV light in the "UVA" region but the unidirectional LED source contained within the cuvette holder means that only small amounts of scattered or reflected rays are emitted from the opening of the cuvette holder. Standard personal protective equipment (laboratory coat, nitrile gloves, polycarbonate safety spectacles) offers sufficient protection, but, as an added precaution, students are instructed that while the LED is activated the cuvette holder should be covered with aluminum foil. They are also advised to switch off the LED when inserting or removing cuvettes.

The aldehydes require no special precautions, but furan is toxic by inhalation and should be handled in a well-ventilated area, preferably a fume hood. Toluene and chloroform are harmful by inhalation. The hazards of the oxetane products have not been formally assessed, so students are advised to assume they are toxic and to handle them with the commensurate precautions.

\section{RESULTS AND DISCUSSION}

\section{LED Photolysis Apparatus}

Although there are a large number of commercial LED suppliers, a balance between ease of assembly, operation and cost-effectiveness was considered. For this purpose, a modular optical apparatus was employed, using LEDs that were pre-mounted with 
integrated heat sinks and stored operating parameters. These LEDs can be attached directly to cuvette holders, thus acting as stand-alone miniature photolysis cells (see Appendix in Supporting Information for diagrams of the assembled apparatus). The modular cuvette holders also have ports for up to four optical devices, which enables future flexibility in experimental design, for example, by the attachment of multiple LEDs for increased irradiation intensity, or spectrophotometers for in situ reaction monitoring.

\section{Kinetics Experiments}

For this component of the program, students performed time-course experiments and processed the data to determine the rate constant. Previous studies have shown that the PB reaction is first-order with respect to each component and second-order overall (eq 1):6a,7

$$
\text { Rate }=k[\text { RCHO }][\text { furan }]
$$

where $k$ is the overall rate constant for the reaction, while [RCHO] and [furan] are the concentrations of the selected aldehyde and furan, respectively. Analysis is simplified to pseudo-first-order kinetics through the use of excess furan ([furan] >> [RCHO]) (eq 2):

$$
\text { Rate }=k_{\mathrm{obs}}[\mathrm{RCHO}]
$$

where $k_{\text {obs }}$ is the observed rate constant assuming [furan] $\approx$ constant (eq 3):

$$
k_{\mathrm{obs}}=k[\text { furan }]
$$

Under pseudo-first-order conditions, the data may be fitted to the integral of eq 2. Incorporation of the Beer-Lambert law modifies this equation to use the UV-Vis absorbance rather than the concentration of the aldehyde (eq 4):

$$
\begin{aligned}
& \text { Rate }=-\frac{\mathrm{d}[\mathrm{RCHO}]}{\mathrm{d} t}=k_{\mathrm{obs}}[\mathrm{RCHO}] \\
& \ln [\mathrm{RCHO}]=-k_{\mathrm{obs}} t+\ln [\mathrm{RCHO}]_{0} \\
& \ln \left(\frac{A_{350}}{\varepsilon_{350} l}\right)=-k_{\mathrm{obs}} t+\ln \left(\frac{A_{350_{0}}}{\varepsilon_{350} l}\right)
\end{aligned}
$$


where $A_{350}$ is the UV-Vis absorbance at $350 \mathrm{~nm}, A_{350_{0}}$ is the absorbance when $t=0, \varepsilon_{350}$ is the molar absorbance coefficient at $350 \mathrm{~nm}$ and $l$ is the cuvette pathlength.

Students used their data to plot graphs of $\ln \left(A_{350}\right)$ against $t$, where the slope of the best-fit line is $-k_{\text {obs }}$ (Figure 1 ).

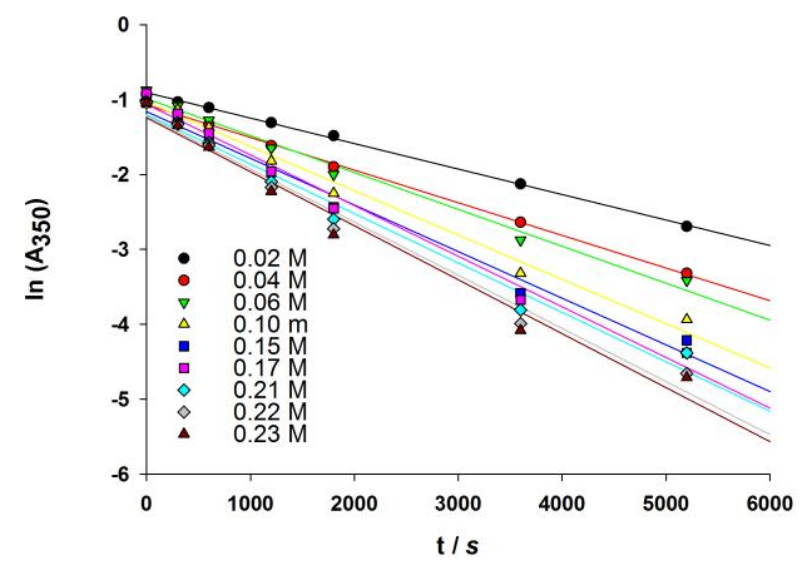

Figure 1. Illustrative example of a graph of $\ln \left(A_{350}\right)$ against time for the consumption of 2-methoxybenzaldehyde 4 in the Paternò-Büchi reaction, for a range of furan concentrations ranging between 0.02 and $0.23 \mathrm{M}$. This graph was plotted using student-generated data.

195

Once the observed rate constants were calculated for a series of furan concentrations, a plot of $k_{\text {obs }}$ against [furan] (eq 3) gives $k$ from the slope of the best-fit line for the benzaldehyde (Figure 2); for the formation of $\mathbf{5 , 6}$ and $\mathbf{7}$, the $k$ values were $7.57 \pm 1.84,4.05 \pm 1.86$ and $32.68 \pm 11.69 \times 10^{-4} \mathrm{M}^{-1} \mathrm{~s}^{-1}$, respectively 


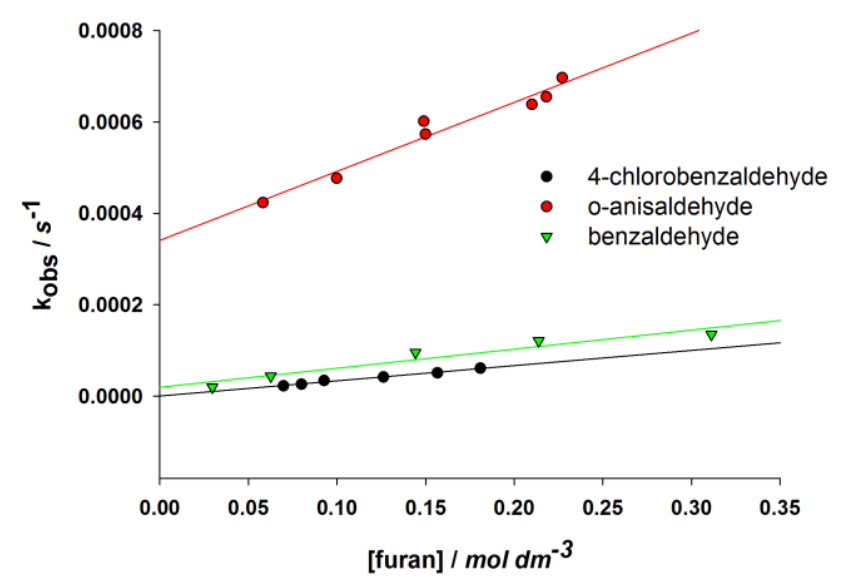

Figure 2. Illustrative example of a graph of $k_{\text {obs }}$ against [furan] for the Paternò-Büchi reaction with 2methoxybenzaldehyde 4, benzaldehyde 2 and 4-chlorobenzaldehyde 3 . This graph was plotted using student-generated data.

Actinometry and Quantum Yield Determination

A further task that students undertook was the determination of the differential quantum yield ${ }^{8}$ for each of the PB reactions. For this purpose, students measured the photon flux delivered by the LED apparatus using chemical actinometry. A number of actinometers have been described in the literature, but the ferrioxalate actinometer was found to be the easiest to implement, as its results are not strongly influenced by temperature or the presence of oxygen. ${ }^{9}$ Using this actinometer, a rate of photon delivery, $q$, of $2.48 \pm 1.07 \times 10^{-8} \mathrm{~mol} \mathrm{~s}^{-1}$ was typically achieved by students.

The differential quantum yield at $365 \mathrm{~nm}, \Phi_{365}$, taking into account the volume of 215 the reaction mixture, can be calculated using eq 5:

$$
\Phi_{365}=\frac{\text { rate of reaction }}{\text { rate of photon absorption }}=\frac{k[\mathrm{RCHO}][\mathrm{furan}] V}{q\left(1-10^{\left.-A_{365}\right)}\right.}
$$

where $V$ is the volume of the reaction mixture and $A_{365}$ is the UV-Vis absorbance at 365 $\mathrm{nm}$. Since $\Phi_{365}$ is dependent on the concentrations of the reactants, the IUPAC recommends that the initial differential quantum yield is reported using the starting concentrations and initial $A_{365}$. At a [furan] of $0.15 \mathrm{M}$, students typically achieved a $\Phi_{365}$ for 5, 6 and $\mathbf{7}$ of $0.107 \pm 0.012,0.061 \pm 0.002$ and $0.385 \pm 0.037$, respectively. Note, 
however, that, since differential quantum yields are dependent on the rate of reaction, the final values that students achieve will depend on the actual concentration of furan they used.

Students compared and contrasted the calculated values of the different benzaldehydes with respect to the electron donating or withdrawing nature of the substituents. The electron donating methoxy substituent resulted in a higher rate constant and quantum yield; while the electron withdrawing chloro substituent gave the opposite effect (see Instructors' Notes in Supporting Information for detailed discussion).

Compound Analysis and Characterization

As an exercise in organic compound characterisation, students undertook the full chemical characterization of the oxetane products. By performing the PB reaction on a larger scale, they isolated sufficient material to enable analysis of the product by NMR and IR spectroscopy to confirm its structure.

The regiochemistry was unambiguously assigned by a diagnostic ${ }^{1} \mathrm{H}-\mathrm{NMR}$ doublet between $6.4-6.6 \mathrm{ppm}$ corresponding to the acetal proton (see representative student ${ }^{1} \mathrm{H}$ NMR spectra in the Supporting Information). The relative exo-stereochemistry was assigned using NOESY NMR experiments. ${ }^{5 b}$ In the case of the benzaldehyde adduct $\mathbf{5}$, it was also possible to determine the stereochemistry by comparison of the ${ }^{1} \mathrm{H}-\mathrm{NMR}$ spectra of the endo- and exo-adducts since the proton that is facing the aromatic ring is shielded by the induced ring current (see Instructors' Notes in Supporting Information).

The students can confirm the purity of the oxetane products from the IR spectra. Here, the strong absorbances corresponding to the carbonyl stretch in the benzaldehyde 245 starting materials are abolished in the resulting products. Since this signal is typically strong and would be apparent even if a small amount of starting material was present in the sample. For example, a carbonyl absorbance peak $\left(\sim 1694 \mathrm{~cm}^{-1}\right)$ is clearly 
observable in the student acquired IR spectra for oxetane $\mathbf{6}$ that was produced from the chlorobenzaldehyde $\mathbf{3}$, where the separation of the product from the starting material is more difficult. This result can also be confirmed by the presence of a signal corresponding to the aldehyde proton in the ${ }^{1} \mathrm{H}$ NMR spectrum (see supporting information for student acquired IR and NMR spectra for this compound).

The data from the MS also serve to support the presence of expected oxetanes. In all cases a peak corresponding to the molecular ion is observed, in addition to peaks corresponding to fragment ions for the (substituted) phenyl ring. In the case of oxetane 6, it is also possible to observe the distinctive chlorine isotopic distribution ([M]: $[\mathrm{M}+2]$, 3:1) for the molecular ion and phenyl fragment, though the latter may be obscured by the presence of other fragment ions.

\section{Student Commentary}

An analysis of the students' written reports and their feedback (based on a formal questionnaire) indicated that students coped well with the experimental work and were able to extract the relevant constants by processing of the collected data. In general, the best students commented that the kinetics calculations were straightforward. The isolation of the oxetanes was generally carried out well by the students for $\mathbf{5}$ and $\mathbf{7}$, with good levels of purities achieved. The purity of the chlorinated oxetane $\mathbf{6}$ generally achieved was lower, primarily due to the students' lower proficiency with column chromatography. Nevertheless, spectroscopic analysis and assignments of the signals was generally successfully carried out by the students with minimal aid by the teaching assistants or instructors.

In regards to the discussion of the results, most students were less able to independently draw together different topics and to generate in-depth rationalisations of their results. For example, relating chemical structures to the observed rates (e.g., why does the electron poor aldehyde react more slowly?), or rationalising the origin of the 
observed regio- and stereochemistry (why is the exo product preferred?). In this regard, guidance from teaching assistants was often needed to help students contextualise their results. Nevertheless, the overall aim of exposing students to a holistic experimental program, where topics taught separately must be combined to rationalise the observed results, was achieved. Furthermore, all students found the LED apparatus easy to operate. In all cases, students were able to employ the apparatus for their experiments with no intervention after just two demonstrations.

\section{CONCLUSIONS AND OVERVIEW}

The implementation of the photochemical Paternò-Büchi reaction in an undergraduate laboratory was described. The LED-based photolysis apparatus was found to be safe, robust and convenient to operate. The chosen modular design also allowed for the easy modification of the apparatus and the experiment was scalable to any size, being limited only by the availability of equipment (photolysis apparatus and UV-Vis spectrophotometer).

This laboratory experiment was deliberately broad-based, covering photochemistry, spectroscopy, organic chemistry and chemical kinetics in order to demonstrate to students how varied subjects are brought to bear in modern chemical practice. Indeed, there is sufficient scope in this basic experimental framework for a range of exercises and problems, such as actinometry and quantum yield determination, as well as organic structure elucidation by MS, IR and NMR spectroscopy.

\section{ASSOCIATED CONTENT}

295 Supporting Information

Student handout; instructor/demonstrator notes (containing full explanation of kinetics analysis, trouble-shooting and developmental notes); appendix containing information on experimental apparatus and reagents; example student-generated NMR and IR spectra. This material is available via the Internet at http://pubs.acs.org. 


\section{AUTHOR INFORMATION}

Corresponding Author

*E-mail: 1.s.wong@manchester.ac.uk

Notes

305 The authors declare no competing financial interest.

\section{ACKNOWLEDGMENT}

Funding for the photochemistry apparatus was provided by the university's Faculty of Engineering and Physical Sciences' Strategic Fund. We are also grateful to Sven P. K. Koehler and Andrew C. Regan for helpful discussions and advice.

\section{REFERENCES}

(1) (a) Iriondo-Alberdi, J.; Greaney, M. F. Photocycloaddition in Natural Product Synthesis. Eur. J. Org. Chem. 2007, 2007 (29), 4801-4815; (b) Bach, T.; Hehn, J. P. Photochemical Reactions as Key Steps in Natural Product Synthesis. Angew. Chem., Int. Ed. 2011, 50 (5), 1000-1045.

(2) (a) Brown, T. M.; Dronsfield, A. T.; Cooksey, C. J.; Crich, D. An Effective and Facile

315 Demonstration of Organic Photochemistry. J. Chem. Educ. 1990, 67 (5), 434-435; (b) Magner, J. T.; Selke, M.; Russell, A. A.; Chapman, O. L. A New Photochemistry Experiment: A Simple 2+2 Photocycloaddition that Poses an Interesting NMR Problem. J. Chem. Educ. 1996, 73 (9), 854856; (c) Ciana, C.-L.; Bochet, C. G. Clean and Easy Photochemistry. Chimia 2007, 61 (10), 650654; (d) Coyle, E. E.; Oelgemoller, M. Micro-photochemistry: Photochemistry in Microstructured

Reactors. The New Photochemistry of the Future? Photochem. Photobiol. Sci. 2008, 7 (11), 13131322.

(3) (a) Ish-Shalom, M.; Fitzpatrick, J. D.; Orchin, M. Quantitative Analysis by Ultraviolet Spectrophotometry: The cis-trans-Stilbene System. J. Chem. Educ. 1957, 34 (10), 496-499; (b) Davy, J. R.; Jessup, P. J.; Reiss, J. A. The Preparation and Photochemistry of Stilbenes. A Synthetic Organic Chemistry Experiment. J. Chem. Educ. 1975, 52 (11), 747-748; (c) BourelleWargnier, F.; Feigenbaum, A.; Muzart, J. $Z$ - and E-Stereoisomerism: An Experiment Using Photochemistry. J. Chem. Educ. 1978, 55 (5), 339.

(4) (a) Nash, E. G. A Novel Photochemistry Experiment Using a Diels-Alder Reaction. J. Chem. Educ. 1974, 51 (9), 619; (b) D'Auria, M.; Racioppi, R. Oxetane Synthesis through the PaternòBüchi Reaction. Molecules 2013, 18 (9), 11384-11428.

(5) (a) Toki, S.; Shima, K.; Sakurai, H. Organic Photochemical Reactions. I. The Synthesis of Substituted Oxetanes by the Photoaddition of Aldehydes to Furans. Bull. Chem. Soc. Jpn. 1965, 38 (5), 760-762; (b) Griesbeck, A. G.; Stadtmüller, S. Regio- und Stereoselektive 
Photocycloadditionen Aromatischer Aldehyde an Furan und 2,3-Dihydrofuran. Chem. Ber. 1990, $123(2), 357-362$.

(6) (a) Toki, S.; Sakurai, H. Kinetic Studies of the Photoreaction of Benzophenone with Furan. Bull. Chem. Soc. Jpn. 1967, 40 (12), 2885-2889; (b) Salem, L.; Rowland, C. The Electronic Properties of Diradicals. Angew. Chem., Int. Ed. Engl. 1972, 11 (2), 92-111; (c) Freilich, S. C.; Peters, K. S. Picosecond Dynamics of the Paterno-Büchi Reaction. J. Am. Chem. Soc. 1985, 107 340 (13), 3819-3822.

(7) D'Auria, M.; Racioppi, R.; Romaniello, G. The Paternò-Büchi Reaction of 2-Furylmethanols. Eur. J. Org. Chem. 2000, 2000 (19), 3265-3272.

(8) Braslavsky, S. E. Glossary of Terms Used in Photochemistry, 3rd Edition (IUPAC Recommendations 2006). Pure Appl. Chem. 2007, 79 (3), 293-465.

(9) (a) Montalti, M.; Credi, A.; Prodi, L.; Gandolfi, M. T. Handbook of Photochemistry; CRC Press: Boca Raton, 2006; (b) Bowman, W. D.; Demas, J. N. Ferrioxalate Actinometry. A Warning on Its Correct Use. J. Phys. Chem. 1976, 80 (21), 2434-2435. 